Vol 4, No 2 (2021): Stadium -Hungarian Journal of Sport Sciences

https://doi.org/10.36439/shjs/2021/2/10557

\title{
THE FREQUENCY OF USE OF EEG AND NEUROFEEDBACK IN SPORT, SYSTEMATIC REVIEW
}

\author{
Eszter Biró, László Balogh \\ University of Debrecen, Institute of Sport Sciences, Debrecen, Hungary, University of Pécs Doctoral School of \\ Health Sciences, Pécs, Hungary
}

\begin{abstract}
Increasing athlete performance is an eternal challenge in the world of sports. The success of the training work performed can be checked by performance diagnostics. Proper brain processing is essential for skill learning and the implementation of effective motor performance. It was important for brain mapping technology to improve the capabilities of imaging devices in order to measure cognitive-motor performance in the field. The primary purpose of this review was to summarize the frequency of applications of EEG and its associated neurofeedback in sport. Examine the differences and characteristics of protocols. Assess whether there is this uniform, standardized protocol for each sport and how often it is used among both elite and amateur athletes. Electroencephalography was initially used most in sports in which the stable setting was followed by only minimal movement. These include sport shooting, archery and golf and baseball. Later, it was possible to analyze more complex movements with EEG, such as cycling. One of the most commonly used techniques is neurofeedback training, but despite some research on the topic, the arena of neurotechnology in sports psychology still exists in its rudimentary form and is constrained by a plethora of technological problems.
\end{abstract}

Keywords: EEG, sport, neurofeedback 


\section{THEORETICAL BACKROUND}

Increasing athlete performance is an eternal challenge in the world of sports. The success of the training work performed can be checked by performance diagnostics. Proper brain processing is essential for skill learning and the implementation of effective motor performance (SEIDEL-MARZI - RAGERT, 2020).

Cognitive neuroscience uses many types of brain imaging methods (e.g., single-cell electrophysiology, functional near-infrared spectroscopy, functional magnetic resonance imaging, magnetoencephalography, EEG) (PARK et al., 2015; PERREY-BESSON, 2018).

The use of electroencephalography (EEG) dates back to the mid-1920s, when Hans Berger made the first human EEG imaging (MILETT, 2001) and has since become one of the best brain imaging procedures (LIN et al., 2021). An relatively inexpensive and easyto-use tool also plays an important role in its application (GAO et al., 2016). This method helps to study the relationship between the brain and behavior (PARK et al., 2015) by recording waves of different frequencies and amplitudes using electrodes placed on the surface of the scalp (SHELLEY-TREMBLAY, 2006; NEIDERMEYER \& LOPEZ DA SILVA, 1999).

Samples are categorized based on frequencies, which is the primary focus of EEG recording (RAMSAY, 2013). In general, four frequency ranges are distinguished, however, since the state of wakefulness dominates in sports, the alpha and beta waves are considered to be of study significance. During an EEG of individuals, both anatomical and physiological, genetic, and psychological factors are able to influence the strength and prevalence of basal alpha levels (BAZANOVA-VERNON, 2013).

Although cognitive processes are at least as important in any sinter of sport as physical activity diagnostics, neurodiagnostics play a very small role in assessing athletes 'performance (SEIDEL-MARZI - RAGERT, 2020). This is because in most cases it is not feasible to analyze sports behavior outside the laboratory due to the robust nature of the devices and the barriers to the sports environment (PARK et al., 2015; PERREY-BESSON, 2018). The EEG cap and the accelerometer placed on the head emitted oscillations with the same properties when walking on a treadmill (CASTERMANS et al., 2014). Overall, one of the major disadvantages of EEG is that it is very sensitive to motion products (SYMEONIDOU et al., 2018), and its spatial resolution can be said to be relatively low (MEHTA-PARASURAMAN, 2013).

Consequently, it was important for brain mapping technology to improve the capabilities of imaging devices in order to measure cognitive-motor performance in the field (PARK et al., 2015; PERREY-BESSON, 2018).

The traditional wet sensory systems of the EEG were not mobile and their preparation time was long, as a result of which it was not possible to apply the sport in practice. With the recent introduction of a wireless, dry EEG system, however, a new gate is opening for real-time measurement in the field in sports (LIN et al., 2021). 
In today's sports education, the development of physical aspects is coordinated with mental training to increase performance (BALCONI et al., 2019). Training methods aimed at optimal performance are predominantly focused on internal processes, emotional acceptance, and self-control (GARDNER-MOORE, 2007).

Recently, one of the most commonly used techniques is neurofeedback training (DOMINGOS et al., 2021). According to Mirifar (2017) and Balconi (2019), "neurofeedback is a psychophysiological technique aimed at raising awareness of the central physiological relationships of internal experiences and teaching adaptive regulation of such relationships and related thinking".

Sports with different aspects use other neurofeedback-based training programs: some that stimulate concentration and mental focus by modifying the sensorimotor rhythm, and some that help to relax and reduce excitement and performance constraints by modulating EEG bands (alpha, theta). anxiety (MIRIFAR et al., 2017; BALCONI et al., 2019).

The effectiveness of the method is measured by the speed and accuracy of the implementation of the planned action (SALEH et al., 2006). It has a beneficial effect on any movement affecting motor performance that requires automatic movements and steep learning curves (SIDHU-COOKE, 2021).

The condensed training protocol increased alpha amplitude more effectively, and there was a significant improvement in the performance of N-back, a strange test following neurofeedback training to assess attention (DOMINGOS et al., 2021) and most parameters of the partial curve (MIKICIN et al. al., 2020).

Neurofeedback can also be a great way to determine and train the optimal level of optimal nervous system activity, arousal, in an individual (NÉMETH-BALOGH, 2021)

Overall, neurofeedback is a promising non-invasive procedure that can be used to develop skills related to self-regulation of brain activity (MIRIFAR et al., 2017). However, further testing and comparison of protocols is needed in the future to better understand how increased alpha waves contribute to more effective athletic performance (DOMINGOS et al., 2021). 


\section{METHODS}

The process for this systematic review was informed by the PRISMA guidelines (MOHER et al., 2009), summarised in Fig. 1.

\section{SEARCH STRATEGIES}

Electronic databases (Pubmed, ScienceDirect, SpringLink, PsycNet, Google Scholar) were searched for with the keyword combinations included "EEG" in conjunction with "sport" and "neurofeedback". The search was restricted to English and Spanish peer-reviewed articles. In the initial search 215 articles were detected. Pursuing this, keywords of the articles were analysed for farther search combinations. Two new search terms were determined and united with the existing terms including "psychophysiological" and "brain-computer interface", and subsequently searched within the databases. A further 39 articles were determined with the new search terms in the five databases, resulting in a total of 254 .

\section{Inclusion and exclusion of studies}

Studies incorporated in this systematic review corresponded to the following criteria: (a) the application of EEG has taken place in the field of sport (i.e., clinical researches were excluded), (b) participant groups contained amateur and professional athletes (i.e., former athletes have been excluded, because we are unable to track their current athletic performance.

\section{Screening articles}

Each article was screened by investigating the title, keywords and abstract based on the inclusion criteria. If there was any indeterminancy over the suitability of an article, this was debated by the first and second author. 
Vol 4, No 2 (2021): Stadium -Hungarian Journal of Sport Sciences

https://doi.org/10.36439/shjs/2021/2/10557
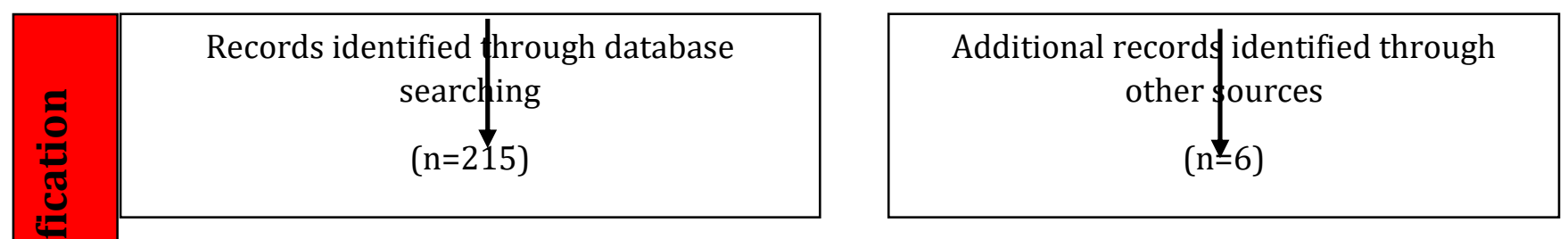

C

Records after duplicates removed

$$
(n=188)
$$
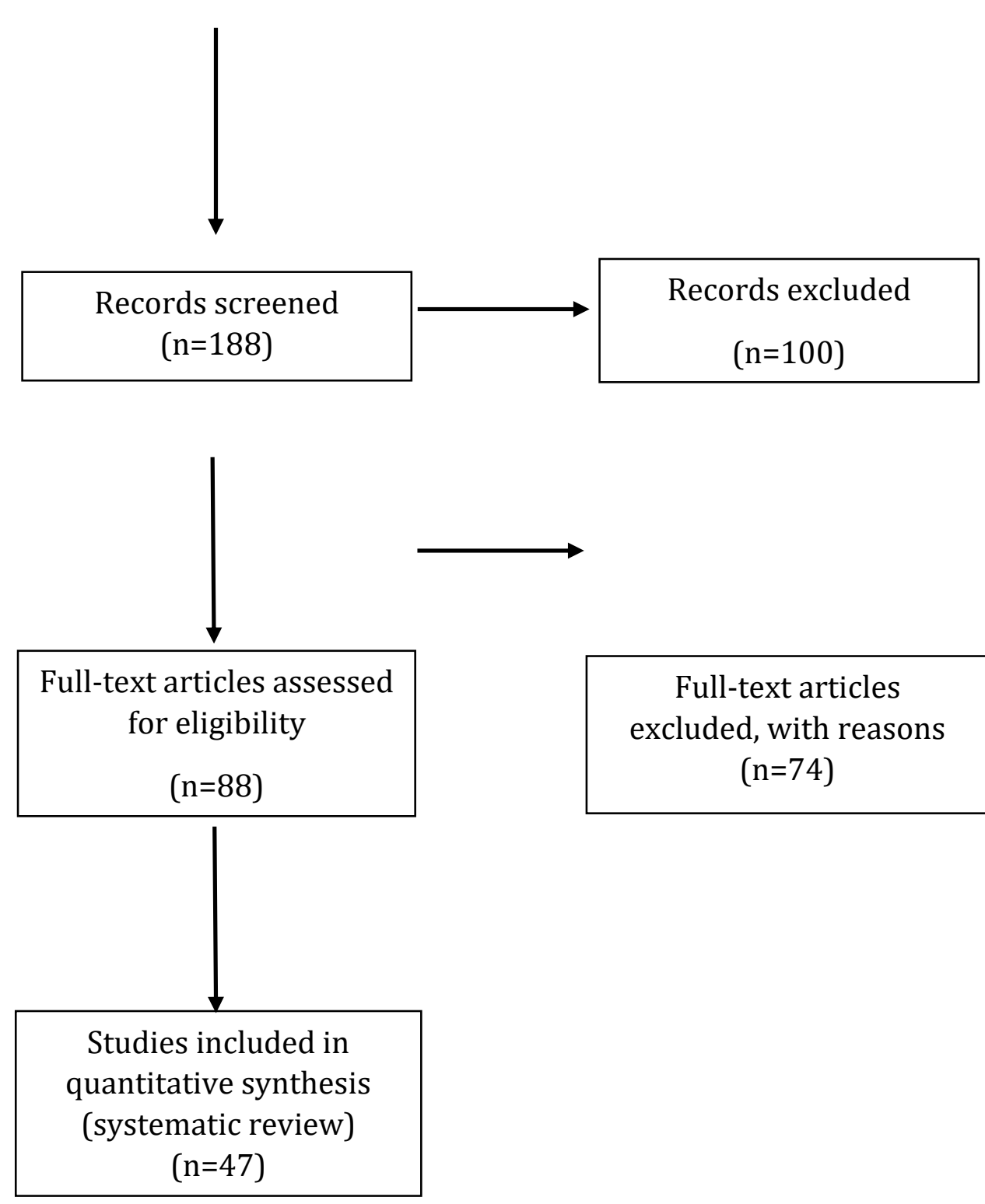

Full-text articles excluded, with reasons $(n=74)$

Fig. 1. : Diagram of PRISMA flow 


\section{RESULTS}

The primary purpose of this review was to summarize the frequency of applications of EEG and its associated neurofeedback in sport. Besides to get the answer which sports are where EEG devices and the neurofeedback procedure are used, a complete review of 90 articles was managed after finding potentially relevant ones based on the examination of titles, keywords and abstracts. In order to determine the suitability of the full-text articles, they were re-examined and evaluated against the above-mentioned inclusion criteria. Most studies were excluded because they were clinical patients who had diseases related to the mind or brain, not athletes $(n=43)$. A total of 47 studies were included in the final analysis. All studies were performed under laboratory conditions $(n=47)$.

After the study, it became clear to us that electroencephalography was initially used most in sports in which the stable setting was followed by only minimal movement. These include sport shooting (DOPPELMAYR et al., 2008; PEREIRA et al., 2018), archery (SALAZAR et al., 1990; Landers et al., 1994) and golf from ball sports (BABILONI et al., 2008). ; REINECKE et al., 2011) and baseball (MURASKIN et al., 2015; PLUTA et al., 2018).

Later, it was possible to analyze more complex movements with EEG, such as cycling in ergometer (LIN et al., 2021).

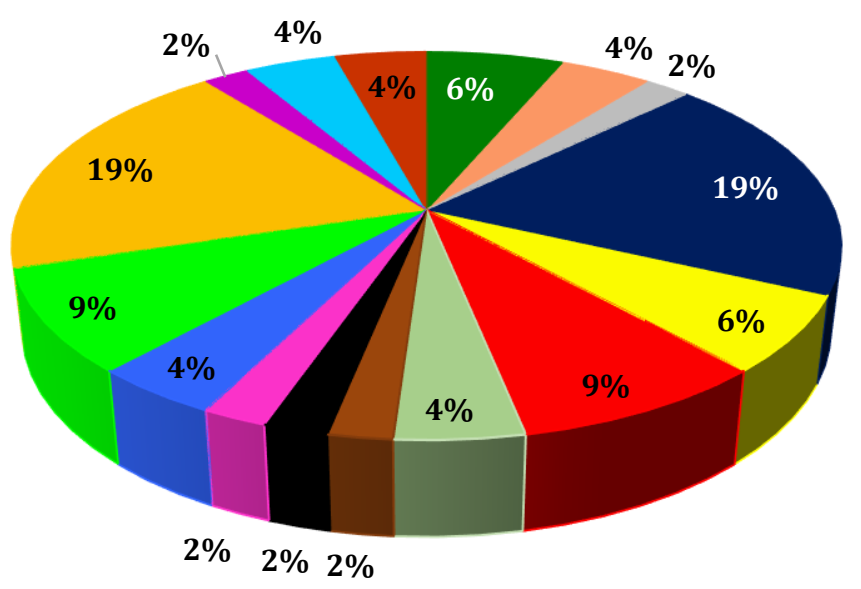

\begin{tabular}{|c|c|c|c|}
\hline Archery & Baseball & Boxing & Cycling \\
\hline Diving & Football & Golf & Gymnastics \\
\hline Ice hockey & Judo & Karate & Running \\
\hline ng & Skiing & Swimming & Throwing games \\
\hline
\end{tabular}

Fig. 2: Percentage of EEG use in sports based on the 47 systematic reviews examined

For the 47 studies examined, we encountered different research goals. Most often, EEG activity was analyzed before, during, and after sports activity $(n=36)$. Within these, there were 3 studies that considered spectral and temporal aspects separately. The second most 
important aspect examined the effect of neurofeedback training (NFT) on athletes ( $\mathrm{n}=8$ ). This was followed by a study of hemodynamic response change $(n=3)$.

One $(n=35)$ or two groups $(n=12)$ were examined in the analyzed articles. The research involved elite athletes $(n=26)$ and we found comparative studies between experts and non-experts $(n=9)$. The remaining studies examined the condition of young athletes $(n=$ 8). In some cases, non-athletes also participated in specific sports tasks $(n=5)$.

In the case of the articles reviewed, it is important to highlight that most studies (65\%) conducted mixed studies using EEG in terms of gender. However, comparing non-mixed research, it can be stated that men (26\%) were more concerned with performance.

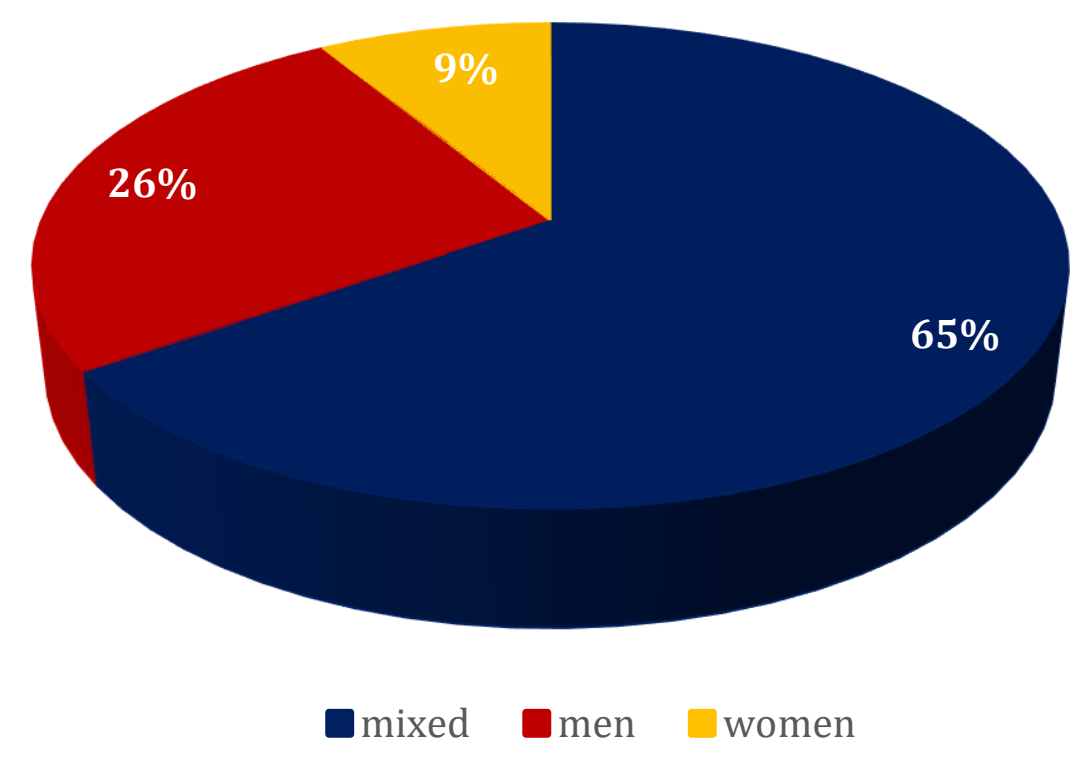

Fig. 3: The proportion of gender distribution in the reviewed articles

The tests could be divided into two groups for the additional tests attached to the EEG. Of the 47 studies examined, 21 did not use additional procedures, and in the other cases other tests were performed $(n=26)$.

The most common of these was electromyography, used in 11 cases. In a few cases ( $\mathrm{n}=$ 4), the so-called EOG (electrooculogram) was also associated with electroencephalography. Measurement of systolic (SBP) and diastolic blood pressure (DBP) appeared in 1-1 cases and HR in 7 cases as an additional study. While the measurement of respiratory parameters as $\mathrm{VO}_{2} \max (n=6)$ and $\mathrm{VCO}_{2}(n=2)$ and Respiration Rate $(n=1)$ also played a role in the laboratory studies.

Anthropometric indices as height was measured $(n=2)$, (FAT) fat percentage $(n=2)$, (LBM) lean body mass $(n=3)$, (TBW) total body water $(n=2)$ were showed using Bioelectrical Impedance Analysis (BIA). In addition, lactate concentration ( $n=3)$, (SC) skin conductance $(n=1)$, (COP) body of center of pressure $(n=1)$, myocardial oxygen consumption index $(n=1)$ were also mentioned parameters during EEG studies. 
Several authors attributed a significant role to the survey in their study $(\mathrm{n}=12)$. Which appeared in the form of questionnaires $(n=8)$ or scales $(n=4)$. The questionnaires were as follows: (BIS / BAS) Behavioral Inhibition and Activation System questionnaire ( $\mathrm{n}=1)$, EHIS (European Health Interview Survey) questionnaire $(\mathrm{n}=1)$ ), Health questionnaire ( $\mathrm{n}$ $=2$ ), (IPAQ) Intern Physical Activity Questionnaire $(n=1)$ and (PTIQ) Post-trial imagery questionnaire $(\mathrm{n}=1)$. The scales used were: BRUNEL Mood Scale $(\mathrm{n}=1)$, Category Ratio Scale $(\mathrm{n}=2)$, (SSCCS) State Self-Control Capacity Scale $(\mathrm{n}=1)$ and (VAS) Visual Analogue Scale $(\mathrm{n}=1)$.

Based on the results of several studies, it can be stated that the decrease in cortical activation is closely correlated with excellent performance and higher levels of intelligence (GRABNER et al., 2006), (NEUBAUER et al., 2004). This phenomenon (neural efficiency) is called the psychomotor efficiency hypothesis (HATFIELD et al., 2020). Several studies have found a difference between the athlete group and the control group in the correlation of EEG overall energy in motor imaging tasks (GAO et al., 2016), (SALENIUS-HARI, 2006), which collects specific brain networks during decision situations (FINK et al. 2018). As a result, experts perform more effectively than novices (BABILONI et al., 2010), (GRABNER et al., 2006). More specifically, in the eye-closed resting state, athletes produced higher alpha values in the parietal and occipital areas (BABILONI et al., 2010), (BEYER et al., 1990). In general, elevated alpha activity in the left temporal lobe is associated with better performance, however, high alpha activity in both temporal lobes has been shown to show the worst performance (JANELLE-HATFIELD, 2008).

According to some EEG studies, comparing worst and best performance, higher alpha performance activity can be associated with less good performance (LANDERS et al., 1994), while other studies suggest that the best performance measure is increased alpha performance and this increase is related to with increasing skill levels (LOZE et al., 2001). Task-related performance loss (TRPD) indexes cortical activity (DEL PRECIO et al., 2011). Both low and high TRDP showed lower scores in athletes than in non-athlete control groups. This also confirms the hypothesis of neural efficacy that cortical reactivity decreases when the eyes open at rest (DEL PRECIO et al., 2011).

Numerous studies have demonstrated that neurofeedback training is already effective in various fields of health science, including reduction of fear, increased ability to concentrate, and improved physical coordination (Kerick et al., 2004),(Besserve et al., 2008). In addition, our analysis revealed that in many sports (martial arts, hockey, skating, basketball, snowboarding), sports performance improves as a result of neurofeedback training (MIKICIN et al., 2020). We did not find a uniform neurofeedback training system for different sports, however, different programs aim to keep the subject calm even under stress and focus attention on the goals of the task (MIRIFAR et al., 2017). As a result of our work, it can be stated that despite some studies on neurofeedback training, this field is still very rudimentary and many articles highlight technical 
Vol 4, No 2 (2021): Stadium -Hungarian Journal of Sport Sciences

https://doi.org/10.36439/shjs/2021/2/10557

difficulties, but may be a suitable option in the field of sports learning and training in the future (CAMILLE et al., 2020).

\begin{tabular}{|c|c|c|c|c|c|c|c|c|c|}
\hline AUTHOR & $\begin{array}{l}\text { TITLE OF THE } \\
\text { STUDY }\end{array}$ & $\begin{array}{l}\text { NUMBER } \\
\text { OF } \\
\text { SUBJECTS }\end{array}$ & GENDER & SPORT & $\begin{array}{l}\text { TERRITORIAL } \\
\text { DEFINITION }\end{array}$ & AGE & CLASS & $\begin{array}{c}\text { METHODOL } \\
\text { OGY }\end{array}$ & AIM OF THE STUDY \\
\hline $\begin{array}{c}\text { Duru, A. D., \& } \\
\text { Assem, M., } 2018\end{array}$ & \begin{tabular}{|} 
Investigating neural \\
efficiency of elite \\
karate athletes \\
during a mental \\
arithmetic task using \\
EEG
\end{tabular} & 10 & $\begin{array}{c}1 \text { woman, } \\
9 \text { men }\end{array}$ & $\begin{array}{c}\text { individual: } \\
\text { karate }\end{array}$ & $\begin{array}{l}\text { Marmara } \\
\text { University, } \\
\text { Turkey }\end{array}$ & $\begin{array}{c}\text { students } \\
\text { of } \\
\text { university }\end{array}$ & $\begin{array}{c}\text { medals in } \\
\text { national and } \\
\text { international } \\
\text { competitions }\end{array}$ & \multirow{4}{*}{$\begin{array}{c}\text { experimental } \\
\text { procedure }\end{array}$} & $\begin{array}{c}\text { the involvement of } \\
\text { neural efficiency in a } \\
\text { cognitive task in } \\
\text { elite athletes using } \\
\text { EEG }\end{array}$ \\
\hline $\begin{array}{l}\text { Hillman, C. H., } \\
\text { Apparies, R. J., } \\
\text { Janelle, C. M., \& } \\
\text { Hatfield, B. D., } \\
2000\end{array}$ & $\begin{array}{l}\text { An electrocortical } \\
\text { comparison of } \\
\text { executed and } \\
\text { rejected shots in } \\
\text { skilled marksmen }\end{array}$ & 12 & men & $\begin{array}{c}\text { individual: } \\
\text { shooting }\end{array}$ & $\begin{array}{c}\text { members of the } \\
\text { National Rifle } \\
\text { Association in } \\
\text { Fairfax, US }\end{array}$ & $\begin{array}{c}\text { mean: } 34 \\
\text { years }\end{array}$ & \begin{tabular}{|c|} 
expert ranking \\
by National \\
Rifle \\
Association \\
(90-95\% \\
accuracy) \\
\end{tabular} & & $\begin{array}{c}\text { the EEG activity } \\
\text { during preparatory } \\
\text { period }\end{array}$ \\
\hline $\begin{array}{l}\text { Mikicin, M., Mróz, } \\
\text { A., Karczewska- } \\
\text { Lindinger, M., } \\
\text { Malinowska, K., } \\
\text { Mastalerz, A., \& } \\
\text { Kowalczyk, M., } \\
2020\end{array}$ & $\begin{array}{c}\text { Effect of the } \\
\text { Neurofeedback-EEG } \\
\text { Training During } \\
\text { Physical Exercise on } \\
\text { the Range of Mental } \\
\text { Work Performance } \\
\text { and Individual } \\
\text { Physiological } \\
\text { Parameters in } \\
\text { Swimmers }\end{array}$ & 7 & - & $\begin{array}{c}\text { individual: } \\
\text { swimming }\end{array}$ & \begin{tabular}{|} 
Józef Piłsudski \\
University of \\
Physical \\
Education, \\
Warsaw, \\
Poland
\end{tabular} & $\begin{array}{l}18-25 \\
\text { years }\end{array}$ & - & & $\begin{array}{c}\text { demonstrate the } \\
\text { effects of the } \\
\text { Neurofeedback-EEG } \\
\text { training during } \\
\text { physical exercise on } \\
\text { the improvements in } \\
\text { mental work } \\
\text { performance and } \\
\text { physiological } \\
\text { parameters }\end{array}$ \\
\hline $\begin{array}{c}\text { Carius, D., Seidel- } \\
\text { Marzi, O., } \\
\text { Kaminski, E., } \\
\text { Lisson, N., \& } \\
\text { Ragert, P., } 2020\end{array}$ & $\begin{array}{c}\text { Characterizing } \\
\text { hemodynamic } \\
\text { response alterations } \\
\text { during basketball } \\
\text { dribbling }\end{array}$ & 23 & $\begin{array}{c}12 \\
\text { women, } \\
11 \text { men }\end{array}$ & $\begin{array}{c}\text { team: } \\
\text { basketball }\end{array}$ & $\begin{array}{l}\text { University of } \\
\text { Leipzig, } \\
\text { Germany }\end{array}$ & $\left|\begin{array}{c}24.61 \pm \\
0.47 \text { years }\end{array}\right|$ & - & & \begin{tabular}{|c} 
compare \\
hemodynamic \\
response alterations \\
during a BSDT with \\
varying levels of \\
complexity including \\
performance (with \\
dominant, non- \\
dominant, both \\
hands)
\end{tabular} \\
\hline $\begin{array}{c}\text { Ludyga, S., } \\
\text { Gronwald, T., \& } \\
\text { Hottenrott, K., } \\
2016\end{array}$ & $\begin{array}{l}\text { The Athlete's Brain: } \\
\text { Cross-Sectional } \\
\text { Evidence for Neural } \\
\text { Efficiency during } \\
\text { Cycling Exercise }\end{array}$ & 29 & $\begin{array}{c}11 \\
\text { women, } \\
18 \text { men }\end{array}$ & $\begin{array}{c}\text { individual: } \\
\text { cyclism, } \\
\text { triathlon }\end{array}$ & $\begin{array}{c}\text { Basel, } \\
\text { Switzerland }\end{array}$ & $\begin{array}{l}18-35 \\
\text { years }\end{array}$ & $\begin{array}{c}\text { from local } \\
\text { sport clubs (4 } \\
\text { hours training } \\
\text { per week) }\end{array}$ & $\begin{array}{l}\text { cross- } \\
\text { sectional } \\
\text { trial }\end{array}$ & $\begin{array}{c}\text { examine brain } \\
\text { cortical activity at } \\
\text { rest and during } \\
\text { exercise between } \\
\text { cyclists of higher } \\
\text { and lower maximal } \\
\text { oxygen } \\
\text { consumption }\end{array}$ \\
\hline $\begin{array}{c}\text { di Fronso, S., } \\
\text { Tamburro, G., } \\
\text { Robazza, C., } \\
\text { Bortoli, L., } \\
\text { Comani, S., \& } \\
\text { Bertollo, M., } \\
2018\end{array}$ & $\begin{array}{l}\text { Focusing Attention } \\
\text { on Muscle Exertion } \\
\text { Increases EEG } \\
\text { Coherence in an } \\
\text { Endurance Cycling } \\
\text { Task }\end{array}$ & 11 & $\begin{array}{c}4 \text { women, } \\
7 \text { men }\end{array}$ & $\begin{array}{c}\text { individual: } \\
\text { cyclism }\end{array}$ & \begin{tabular}{|c|} 
"G. d'Annunzio" \\
University of \\
Chieti-Pescara, \\
Chieti, Italy
\end{tabular} & $\left|\begin{array}{c}24,29 \pm \\
4,91 \text { years }\end{array}\right|$ & $\begin{array}{l}2 \text { elite, } 2 \\
\text { amateur, } 7 \\
\text { novice }\end{array}$ & & $\begin{array}{l}\text { examine EEG } \\
\text { coherence before, } \\
\text { during, and after } \\
\text { time to exhaustion } \\
\text { (TTE) trials in an } \\
\text { endurance cycling } \\
\text { task }\end{array}$ \\
\hline $\begin{array}{l}\text { Broelz, E.K., Enck, } \\
\text { P., Niess, A.M. } \\
\text { Schneeweiss, P., } \\
\text { Wolf, S., Weimer, } \\
\text { K., } 2019\end{array}$ & $\begin{array}{l}\text { The neurobiology of } \\
\text { placebo effects in } \\
\text { sports: EEG frontal } \\
\text { alpha asymmetry } \\
\text { increases in } \\
\text { response to a } \\
\text { placebo ergogenic } \\
\text { aid }\end{array}$ & 19 & men & $\begin{array}{c}\text { individual: } \\
\text { cyclism } \\
\text { (mountain } \\
\text { bike, road } \\
\text { bike, } \\
\text { triathlon) }\end{array}$ & $\begin{array}{l}\text { University } \\
\text { Hospital } \\
\text { Tuebingen, } \\
\text { Germany }\end{array}$ & $\mid \begin{array}{c}18-50 \\
\text { years, } \\
37,26 \pm \\
9,81 \text { years }\end{array}$ & $\begin{array}{l}\text { competitive } \\
\text { cyclist (3 } \\
\text { weekly } \\
\text { training } \\
\text { during } \\
\text { competition } \\
\text { season, } 3 \\
\text { competitions } \\
\text { per year) }\end{array}$ & $\begin{array}{c}\text { experimental } \\
\text { procedure }\end{array}$ & $\begin{array}{l}\text { test the increase in } \\
\text { FAA (frontal alpha } \\
\text { assymetry) in } \\
\text { response to a PEA } \\
\text { (placebo ergogenic } \\
\text { aid) and explore the } \\
\text { role of the } \\
\text { behavioural } \\
\text { activation and } \\
\text { inhibition system } \\
\text { (BAS/BIS) }\end{array}$ \\
\hline $\begin{array}{l}\text { Jain, S., Gourab, } \\
\text { K., Schindler- } \\
\text { Ivens, S., \& } \\
\text { Schmit, B. D, }\end{array}$ & $\begin{array}{l}\text { EEG during pedaling: } \\
\text { evidence for cortical } \\
\text { control of locomotor } \\
\text { tasks. Clinical }\end{array}$ & 10 & - & $\begin{array}{c}\text { individual: } \\
\text { cyclism }\end{array}$ & $\begin{array}{c}\text { Marquette } \\
\text { University, } \\
\text { Milwaukee, } \\
\text { Wisconsin, US }\end{array}$ & $\begin{array}{l}22-32 \\
\text { years }\end{array}$ & - & & $\begin{array}{c}\text { characterize the } \\
\text { brain electrical } \\
\text { activity during } \\
\text { pedaling in a }\end{array}$ \\
\hline
\end{tabular}


Vol 4, No 2 (2021): Stadium -Hungarian Journal of Sport Sciences

https://doi.org/10.36439/shjs/2021/2/10557

\begin{tabular}{|c|c|c|c|c|c|c|c|c|c|}
\hline AUTHOR & $\begin{array}{l}\text { TITLE OF THE } \\
\text { STUDY }\end{array}$ & \begin{tabular}{|c|} 
NUMBER \\
OF \\
SUBJECTS
\end{tabular} & GENDER & SPORT & \begin{tabular}{|} 
TERRITORIAL \\
DEFINITION
\end{tabular} & AGE & CLASS & \begin{tabular}{|} 
METHODOL \\
OGY
\end{tabular} & AIM OF THE STUDY \\
\hline \begin{tabular}{|c|} 
Enders, H., \\
Cortese, F., \\
Maurer, .., \\
Baltich, J., \\
Protzner, A. B., \& \\
Nigg, B. M., 2016
\end{tabular} & $\begin{array}{c}\text { Changes in cortical } \\
\text { activity measured } \\
\text { with EEG during a } \\
\text { high-intensity cycling } \\
\text { exercise }\end{array}$ & 10 & men & $\begin{array}{c}\text { individual: } \\
\text { cyclism }\end{array}$ & $\mid \begin{array}{c}\text { University of } \\
\text { Calgary, Canada }\end{array}$ & $\begin{array}{c}27,5 \pm 5,6 \\
\text { years }\end{array}$ & - & $\begin{array}{c}\text { experimental } \\
\text { procedure }\end{array}$ & \begin{tabular}{|c} 
investigate the \\
effects of a high- \\
intensity cycling \\
exercise on changes \\
in spectral and \\
temporal aspects of \\
EEG
\end{tabular} \\
\hline $\begin{array}{c}\text { Mottola, F., } \\
\text { Blanchfield, A., } \\
\text { Hardy, J., Cooke, } \\
\text { A., } 2021\end{array}$ & $\begin{array}{l}\text { EEG neurofeedback } \\
\text { improves cycling } \\
\text { time to exhaustion }\end{array}$ & 40 & $\begin{array}{c}14 \\
\text { women, } \\
26 \text { men }\end{array}$ & $\begin{array}{c}\text { individual: } \\
\text { cyclism }\end{array}$ & \begin{tabular}{c|} 
Bangor \\
University, UK
\end{tabular} & $\begin{array}{l}18-45 \\
\text { years }\end{array}$ & $\begin{array}{l}\text { recruited from } \\
\text { university and } \\
\text { local clubs }\end{array}$ & $\begin{array}{c}\text { randomized } \\
\text { between- } \\
\text { groups } \\
\text { design }\end{array}$ & $\begin{array}{c}\text { examine an EEG } \\
\text { neurofeedback } \\
\text { intervention for } \\
\text { whole-body } \\
\text { endurance } \\
\text { performance }\end{array}$ \\
\hline $\begin{array}{l}\text { Janelle, C. M., } \\
\text { Hillman, C. H., } \\
\text { Apparies, R. J., } \\
\text { Murray, N. P., } \\
\text { Meili, L., Fallon, } \\
\text { E. A., Hatfield, B., } \\
2000\end{array}$ & $\begin{array}{c}\text { Expertise } \\
\text { differences in } \\
\text { cortical activation } \\
\text { and gaze behavior } \\
\text { during rifle shooting }\end{array}$ & 25 & - & $\begin{array}{c}\text { individual: } \\
\text { shooting }\end{array}$ & US & $\begin{array}{l}13-62 \\
\text { years }\end{array}$ & $\begin{array}{c}\text { experts, } \\
\text { nonexperts }\end{array}$ & & \begin{tabular}{|c|} 
examine whether \\
variability in gaze \\
behavior and \\
cortical activation \\
would differentiate \\
expert and \\
nonexpert small- \\
bore rifle shooters
\end{tabular} \\
\hline $\begin{array}{c}\text { Babiloni, C., Del } \\
\text { Percio, C., } \\
\text { Iacoboni, M., } \\
\text { Infarinato, F., } \\
\text { Lizio, R., } \\
\text { Marzano, N., } \\
\text { Crespi, G., Dassù, } \\
\text { F., Pirritano, M., } \\
\text { Gallamini, M., \& } \\
\text { Eusebi, F, 2008 }\end{array}$ & $\begin{array}{l}\text { Golf putt outcomes } \\
\text { are predicted by } \\
\text { sensorimotor } \\
\text { cerebral EEG } \\
\text { rhythms }\end{array}$ & 12 & $\begin{array}{l}5 \text { women, } \\
7 \text { men }\end{array}$ & $\begin{array}{c}\text { individual: } \\
\text { golf }\end{array}$ & \begin{tabular}{|c|} 
I Medical \\
School, \\
University of \\
Rome \\
'Sapienza', Italy
\end{tabular} & $\begin{array}{l}16-25 \\
\text { years }\end{array}$ & $\begin{array}{c}\text { golfers in } \\
\text { national, } \\
\text { international } \\
\text { competitions }\end{array}$ & $\begin{array}{c}\text { experimental } \\
\text { procedure }\end{array}$ & $\begin{array}{l}\text { investigated how the } \\
\text { sensorimotor alpha } \\
\text { and beta rhythms } \\
\text { were related to } \\
\text { upright balance and } \\
\text { fine arm and hand } \\
\text { motor control } \\
\text { during the putts of } \\
\text { expert golfer }\end{array}$ \\
\hline $\begin{array}{l}\text { García-Monge, A., } \\
\text { Rodríguez- } \\
\text { Navarro, H., } \\
\text { González-Calvo, } \\
\text { G., \& Bores- } \\
\text { García, D., 2020 }\end{array}$ & $\begin{array}{l}\text { Brain Activity during } \\
\text { Different Throwing } \\
\text { Games: EEG } \\
\text { Exploratory Study }\end{array}$ & 8 & $\begin{array}{c}4 \text { women, } \\
4 \text { men }\end{array}$ & throwing & $\begin{array}{l}\text { University of } \\
\text { Valladolid, } \\
\text { Spain }\end{array}$ & $\begin{array}{c}7,2 \pm 0,19 \\
\text { years }\end{array}$ & - & & \begin{tabular}{|c|} 
explore the \\
differences in brain \\
activity in various \\
types of throwing \\
games by making \\
encephalographic \\
records \\
\end{tabular} \\
\hline $\begin{array}{l}\text { Schneider, C., } \\
\text { Pereira, M., } \\
\text { Tonin, L., \& } \\
\text { Millán, J., 2020 }\end{array}$ & $\begin{array}{c}\text { Real-time EEG } \\
\text { Feedback on Alpha } \\
\text { Power Lateralization } \\
\text { Leads to Behavioral } \\
\text { Improvements in a } \\
\text { Covert Attention } \\
\text { Task }\end{array}$ & 14 & $\begin{array}{l}7 \text { women, } \\
7 \text { men }\end{array}$ & - & $\begin{array}{c}\text { Geneva, } \\
\text { Switzerland }\end{array}$ & $\begin{array}{c}23 \pm 1,52 \\
\text { years }\end{array}$ & - & $\begin{array}{l}\text { cross-over } \\
\text { sham- } \\
\text { controlled } \\
\text { study }\end{array}$ & $\begin{array}{c}\text { evaluate the impact } \\
\text { of a new EEG-based } \\
\text { closed-loop } \\
\text { feedback paradigm } \\
\text { on CVSA }\end{array}$ \\
\hline \begin{tabular}{|} 
Jeunet, C., Tonin, \\
L., Albert, L., \\
Chavarriaga, R., \\
Bideau, B., \\
Argelaguet, F., \\
Millán, J., \\
Lécuyer, A., \& \\
Kulpa, R., 2020
\end{tabular} & $\begin{array}{c}\text { Uncovering EEG } \\
\text { Correlates of Covert } \\
\text { Attention in Soccer } \\
\text { Goalkeepers: } \\
\text { Towards Innovative } \\
\text { Sport Training } \\
\text { Procedures }\end{array}$ & 17 & $\begin{array}{c}2 \text { women, } \\
15 \text { men }\end{array}$ & $\begin{array}{c}\text { team: } \\
\text { soccer } \\
\text { goalkeeper }\end{array}$ & Switzerland & $\begin{array}{c}21,4 \pm 5,3 \\
\text { years }\end{array}$ & & & \begin{tabular}{|c} 
investigate \\
neurophysiological \\
(EEG) correlates of \\
Covert Visual Spatial \\
Attention (CVSA) \\
that could be used \\
for NF training \\
procedures \\
dedicated to the \\
improvement of \\
soccer goalkeepers' \\
performances
\end{tabular} \\
\hline \begin{tabular}{|c|} 
Pluta, A., \\
Williams, c. C., \\
Binsted, G., \\
Hecker, K. G., \& \\
Krigolson, O. E., \\
2018
\end{tabular} & $\begin{array}{l}\text { Chasing the zone: } \\
\text { Reduced beta power } \\
\text { predicts baseball } \\
\text { batting performance }\end{array}$ & 67 & men & $\begin{array}{l}\text { team: } \\
\text { baseball }\end{array}$ & $\begin{array}{c}\text { Vancouver and } \\
\text { Victoria, British } \\
\text { Columbia, } \\
\text { Canada }\end{array}$ & $\begin{array}{c}18,2 \pm 1,5 \\
\text { years }\end{array}$ & & $\begin{array}{c}\text { experimental } \\
\text { procedure }\end{array}$ & \begin{tabular}{|c|} 
examine whether or \\
not pre- \\
performance EEG \\
power predicted \\
ensuing \\
performance of an \\
externally-paced \\
motor skill in \\
baseball batting \\
\end{tabular} \\
\hline $\begin{array}{r}\text { Muraskin, J., } \\
\text { Sherwin, J., \& } \\
\text { Sajda, P., } 2015\end{array}$ & $\begin{array}{l}\text { Knowing when not } \\
\text { to swing: EEG } \\
\text { evidence that } \\
\text { enhanced perception- } \\
\text { action coupling } \\
\text { underlies baseball } \\
\text { batter expertise }\end{array}$ & 19 & - & $\begin{array}{l}\text { team: } \\
\text { baseball }\end{array}$ & \begin{tabular}{|c|} 
Columbia \\
University, New \\
York, US
\end{tabular} & $\begin{array}{c}\text { baseball } \\
\text { players: } \\
19.9 \pm 1.1 \\
\text { years, non } \\
\text { player } \\
\text { novices: } \\
21.2 \pm 1.6 \\
\text { years }\end{array}$ & Division I. & & $\begin{array}{c}\text { analyze neural } \\
\text { preparatory signals } \\
\text { of alpha power and } \\
\text { the contingent } \\
\text { negative variation to } \\
\text { compare the pre-- } \\
\text { stimulus responses } \\
\text { of the experts to the } \\
\text { novices }\end{array}$ \\
\hline $\begin{array}{c}\text { Wilson, V. E., } \\
\text { Dikman, Z., Bird, } \\
\text { E. I., Williams, J. } \\
\text { M., Harmison, R., } \\
\text { Shaw-Thornton, } \\
\text { L., \& Schwartz, G. } \\
\text { E., 2016 }\end{array}$ & $\begin{array}{l}\text { EEG Topographic } \\
\text { Mapping of Visual } \\
\text { and Kinesthetic } \\
\text { Imagery in } \\
\text { Swimmers }\end{array}$ & 36 & $\begin{array}{c}19 \\
\text { women, } \\
17 \text { men }\end{array}$ & $\begin{array}{l}\text { individual: } \\
\text { swimming }\end{array}$ & $\begin{array}{l}\text { University of } \\
\text { Arizona, } \\
\text { Tucson, USA }\end{array}$ & $\begin{array}{c}28 \pm 8,9 \\
\text { years }\end{array}$ & Division I & & \begin{tabular}{|c|} 
investigate \\
differences in QEEG \\
measures between \\
kinesthetic and \\
visual imagery of a \\
$100-\mathrm{m}$ swim
\end{tabular} \\
\hline
\end{tabular}


Vol 4, No 2 (2021): Stadium -Hungarian Journal of Sport Sciences

https://doi.org/10.36439/shjs/2021/2/10557

\begin{tabular}{|c|c|c|c|c|c|c|c|c|c|}
\hline AUTHOR & $\begin{array}{l}\text { TITLE OF THE } \\
\text { STUDY }\end{array}$ & $\begin{array}{l}\text { NUMBER } \\
\text { OF } \\
\text { SUBJECTS } \\
\end{array}$ & GENDER & SPORT & \begin{tabular}{|c|} 
TERRITORIAL \\
DEFINITION
\end{tabular} & AGE & CLASS & $\underset{\text { OGY }}{\text { METHODOL }}$ & AIM OF THE STUDY \\
\hline $\begin{array}{l}\text { Beyer, L., Weiss, } \\
\text { T., Hansen, E., } \\
\text { Wolf, A., \& Seidel, } \\
\text { A., } 1990\end{array}$ & $\begin{array}{c}\text { Dynamics of central } \\
\text { nervous activation } \\
\text { during motor } \\
\text { imagination }\end{array}$ & 8 & - & $\begin{array}{l}\text { individual: } \\
\text { swimming }\end{array}$ & $\begin{array}{c}\text { University of } \\
\text { Jena, Germany }\end{array}$ & - & - & \multirow{9}{*}{$\begin{array}{c}\text { experimental } \\
\text { procedure }\end{array}$} & $\begin{array}{l}\text { detect changes } \\
\text { within both of the } \\
\text { last groups of CNS } \\
\text { activation during } \\
\text { motor imagination }\end{array}$ \\
\hline $\begin{array}{c}\text { Lin, C. T., King, J. } \\
\text { T., John, A. R., } \\
\text { Huang, K. C., Cao, } \\
\text { Z., \& Wang, Y. K., } \\
2021\end{array}$ & $\begin{array}{c}\text { The Impact of } \\
\text { Vigorous Cycling } \\
\text { Exercise on Visual } \\
\text { Attention: A Study } \\
\text { With the BR8 } \\
\text { Wireless Dry EEG } \\
\text { System }\end{array}$ & 10 & men & $\begin{array}{c}\text { individual: } \\
\text { cyclism }\end{array}$ & \begin{tabular}{|c|} 
National Chiao \\
Tung \\
University, \\
Taiwan
\end{tabular} & $\begin{array}{c}23,5 \pm 1,5 \\
\text { years }\end{array}$ & - & & $\begin{array}{c}\text { prove that wireless } \\
\text { dry EEG devices can } \\
\text { open up real-time } \\
\text { measurement of } \\
\text { athletes 'cognitive } \\
\text { functions outside } \\
\text { the laboratory }\end{array}$ \\
\hline $\begin{array}{c}\text { Babiloni, C., } \\
\text { Marzano, N., } \\
\text { Iacoboni, M., } \\
\text { Infarinato, F., } \\
\text { Aschieri, P., } \\
\text { Buffo, P., Cibelli, } \\
\text { G., Soricelli, A., } \\
\text { Eusebi, F., \& Del } \\
\text { Percio, C., } 2010\end{array}$ & $\begin{array}{c}\text { Resting state cortical } \\
\text { rhythms in athletes: } \\
\text { a high-resolution } \\
\text { EEG study }\end{array}$ & 74 & $\begin{array}{c}32 \\
\text { women, } \\
52 \text { men }\end{array}$ & $\begin{array}{c}\text { individual: } \\
\text { karate }\end{array}$ & Italy & $\begin{array}{c}\text { elite: } 24,8 \\
\pm 1,1 ; \\
\text { amateur: } \\
23 \pm 1 ; \\
\text { non- } \\
\text { athletes: } \\
26 \pm 0,9 \\
\text { years }\end{array}$ & \begin{tabular}{|c} 
Italian national \\
team, amateur \\
karate athletes
\end{tabular} & & $\begin{array}{c}\text { the amplitude of } \\
\text { resting state cortical } \\
\text { EEG rhythms } \\
\text { (especially alpha, } \\
\text { 8-12 Hz) was higher } \\
\text { in elite athletes } \\
\text { compared with } \\
\text { amateur athletes } \\
\text { and non-athletes }\end{array}$ \\
\hline $\begin{array}{c}\text { Pereira, J., } \\
\text { Sburlea, A.I. \& } \\
\text { Müller-Putz, G.R., } \\
2018\end{array}$ & $\begin{array}{l}\text { EEG patterns of self- } \\
\text { paced movement } \\
\text { imaginations } \\
\text { towards externally- } \\
\text { cued and internally- } \\
\text { selected targets }\end{array}$ & 15 & $\left|\begin{array}{c}7 \text { women, } \\
8 \text { men }\end{array}\right|$ & & $\begin{array}{l}\text { Medical } \\
\text { University of } \\
\text { Graz, Austria }\end{array}$ & $\begin{array}{l}23 \pm 2 \\
\text { years }\end{array}$ & - & & \begin{tabular}{|c|} 
investigate the \\
neurophysiological \\
signature of the \\
interacting \\
processes which \\
lead to a single \\
reach-and-grasp \\
movement \\
imagination \\
\end{tabular} \\
\hline $\begin{array}{l}\text { Doppelmayr, M., } \\
\text { Finkenzeller, T., } \\
\text { \& Sauseng, P., } \\
2008\end{array}$ & $\begin{array}{l}\text { Frontal midline theta } \\
\text { in the pre-shot phase } \\
\text { of rifle shooting: } \\
\text { differences between } \\
\text { experts and novices }\end{array}$ & 18 & - & $\begin{array}{c}\text { individual: } \\
\text { rifle } \\
\text { shooting }\end{array}$ & $\begin{array}{l}\text { Salzburg, } \\
\text { Austria }\end{array}$ & \begin{tabular}{|c|} 
novices: \\
$21-32,7$ \\
years ; \\
experts: \\
$22,6-34,6$ \\
years
\end{tabular} & \begin{tabular}{|l|} 
Salzburg rifle \\
shooting team
\end{tabular} & & $\begin{array}{c}\text { investigate the time } \\
\text { course of frontal } \\
\text { midline theta during } \\
\text { the aimin period in } \\
\text { rifle shooting }\end{array}$ \\
\hline $\begin{array}{l}\text { Haufler, A. J., } \\
\text { Spalding, T. W., } \\
\text { Santa Maria, D. } \\
\text { L., \& Hatfield, B. } \\
\text { D., } 2000\end{array}$ & $\begin{array}{c}\text { Neuro-cognitive } \\
\text { activity during a self- } \\
\text { paced visuospatial } \\
\text { task: comparative } \\
\text { EEG profiles in } \\
\text { marksmen and } \\
\text { novice shooters }\end{array}$ & 15 & $\begin{array}{c}2 \text { women, } \\
13 \text { men }\end{array}$ & $\begin{array}{c}\text { individual: } \\
\text { shooting }\end{array}$ & $\begin{array}{c}\text { University of } \\
\text { West Virginia, } \\
\text { US }\end{array}$ & $\begin{array}{c}26,5 \pm \\
11,1 \text { years }\end{array}$ & \begin{tabular}{|c|} 
competitors in \\
national, \\
international \\
championship \\
$\mathrm{s}$
\end{tabular} & & \begin{tabular}{|c} 
examine the \\
relationship \\
between skill level \\
and cortical \\
activation measured \\
at the left and right \\
frontal, temporal, \\
parietal and \\
occipital sites in \\
marksmen and \\
novices during the \\
preparatory aiming \\
period prior to the \\
trigger pull
\end{tabular} \\
\hline $\begin{array}{c}\text { Fink, A., } \\
\text { Rominger, C., } \\
\text { Benedek, M., } \\
\text { Perchtold, C. M., } \\
\text { Papousek, I., } \\
\text { Weiss, E. M., } \\
\text { Seidel, A., \& } \\
\text { Memmert, D., } \\
2018\end{array}$ & $\begin{array}{l}\text { EEG alpha activity } \\
\text { during imagining } \\
\text { creative moves in } \\
\text { soccer decision- } \\
\text { making situations }\end{array}$ & 43 & men & $\begin{array}{l}\text { team: } \\
\text { soccer }\end{array}$ & $\begin{array}{l}\text { University of } \\
\text { Graz, Austria }\end{array}$ & $\begin{array}{c}24.19 \pm \\
3.16 \text { years }\end{array}$ & \begin{tabular}{|c|} 
the highest \\
soccer league: \\
the third- \\
highest \\
national \\
league
\end{tabular} & & $\begin{array}{l}\text { investigate task- } \\
\text { related changes of } \\
\text { EEG alpha power } \\
\text { while imagining } \\
\text { creative moves in } \\
\text { soccer decision- } \\
\text { making situations }\end{array}$ \\
\hline $\begin{array}{c}\text { Petukhov, I. V., } \\
\text { Glazyrin, A. E., } \\
\text { Gorokhov, A. V., } \\
\text { Steshina, L. A., \& } \\
\text { Tanryverdiev, I. } \\
\text { O., } 2020\end{array}$ & $\begin{array}{l}\text { Being present in a } \\
\text { real or virtual world: } \\
\text { A EEG study }\end{array}$ & 5 & men & - & hiversity of Techr & $\begin{array}{l}50 \pm 5 \\
\text { years }\end{array}$ & & & \begin{tabular}{|c} 
proposes an \\
approach to \\
evaluation and \\
measuring of \\
presence for man- \\
machine interaction \\
in the virtual reality \\
based on \\
electroencephalogra \\
phic data
\end{tabular} \\
\hline $\begin{array}{l}\text { Gao, J., Wang, W., } \\
\text { \& Zhang, J., } 2016\end{array}$ & $\begin{array}{l}\text { Explore } \\
\text { Interregional EEG } \\
\text { Correlations } \\
\text { Changed by Sport } \\
\text { Training Using } \\
\text { Feature Selection }\end{array}$ & 8 & $\begin{array}{c}4 \text { women, } \\
4 \text { men }\end{array}$ & \begin{tabular}{|c|} 
practice \\
full time \\
motor skill
\end{tabular} & China & $\begin{array}{l}20-24 \\
\text { years }\end{array}$ & & & \begin{tabular}{|c} 
explore the EEG \\
correlation patterns \\
changed by sport \\
training (the \\
interregional \\
correlation using \\
the techniques of \\
classification and \\
feature selection)
\end{tabular} \\
\hline
\end{tabular}


Vol 4, No 2 (2021): Stadium -Hungarian Journal of Sport Sciences

https://doi.org/10.36439/shjs/2021/2/10557

\begin{tabular}{|c|c|c|c|c|c|c|c|c|c|}
\hline AUTHOR & $\begin{array}{l}\text { TITLE OF THE } \\
\text { STUDY }\end{array}$ & $\begin{array}{c}\text { NUMBER } \\
\text { OF } \\
\text { SUBJECTS }\end{array}$ & GENDER & SPORT & \begin{tabular}{|c} 
TERRITORIAL \\
DEFINITION
\end{tabular} & AGE & CLASS & $\underset{\text { OGY }}{\text { METHODOL }}$ & AIM OF THE STUDY \\
\hline $\begin{array}{l}\text { Del Percio, C., } \\
\text { Infarinato, F., } \\
\text { Marzano, N., } \\
\text { Iacoboni, M., } \\
\text { Aschieri, P., Lizio, } \\
\text { R., Soricelli, A., } \\
\text { Limatola, C., } \\
\text { Rossini, P. M., \& } \\
\text { Babiloni, C., } \\
2011\end{array}$ & $\begin{array}{c}\text { Reactivity of alpha } \\
\text { rhythms to eyes } \\
\text { opening is lower in } \\
\text { athletes than non- } \\
\text { athletes: a high- } \\
\text { resolution EEG study }\end{array}$ & 26 & women & $\begin{array}{l}\text { individual: } \\
\text { karate }\end{array}$ & $\begin{array}{c}\text { University of } \\
\text { Rome Sapienza, } \\
\text { Italy }\end{array}$ & - & $\begin{array}{c}\text { Italian national } \\
\text { karate team }\end{array}$ & \multirow{11}{*}{$\begin{array}{l}\text { experimental } \\
\text { procedure }\end{array}$} & \begin{tabular}{|} 
tested the \\
hypothesis that \\
compared with non- \\
athletes, elite \\
athletes are \\
characterized by a \\
decrease of cortical \\
reactivity to eyes \\
opening in resting \\
condition
\end{tabular} \\
\hline $\begin{array}{l}\text { Ring, C., Cooke, } \\
\text { A., Kavassanu, M., } \\
\text { McIntyre, D., } \\
\text { Masters, R., 2015 }\end{array}$ & $\begin{array}{c}\text { Investigating the } \\
\text { efficacy of } \\
\text { neurofeedback } \\
\text { training for } \\
\text { expediting expertise } \\
\text { and excellence in } \\
\text { sport }\end{array}$ & 24 & men & $\begin{array}{c}\text { individual: } \\
\text { golf }\end{array}$ & $\begin{array}{c}\text { University of } \\
\text { Birmingham, } \\
\text { UK }\end{array}$ & \begin{tabular}{|c|} 
NFT \\
group: 23 \\
$\pm 5,83$ \\
years, \\
control \\
group: 21 \\
$\pm 2,52$ \\
years
\end{tabular} & & & $\begin{array}{c}\text { examine whether } \\
\text { EEG based } \\
\text { neurofeedback } \\
\text { could be used to } \\
\text { train recreational } \\
\text { golfers to regulate } \\
\text { their brain activity, } \\
\text { expedite skill } \\
\text { acquisition, and } \\
\text { promote robust } \\
\text { performance under } \\
\text { pressure }\end{array}$ \\
\hline $\begin{array}{l}\text { Zhu, F. F., } \\
\text { Poolton, J. M., } \\
\text { Wilson, M. R., } \\
\text { Maxwell, J. P., \& } \\
\text { Masters, R. S., } \\
2011\end{array}$ & \begin{tabular}{|c} 
Neural co-activation \\
as a yardstick of \\
implicit motor \\
learning and the \\
propensity for \\
conscious control of \\
movement
\end{tabular} & 16 & - & $\begin{array}{c}\text { individual: } \\
\text { golf }\end{array}$ & $\begin{array}{c}\text { University of } \\
\text { Hong Kong, } \\
\text { Hongkong }\end{array}$ & $\begin{array}{c}\text { Low: } 21,8 \\
\pm 1,67 \\
\text { years; } \\
\text { High: } 23,3 \\
\pm 3,2 \\
\text { years }\end{array}$ & & & $\begin{array}{c}\text { examine EEG co- } \\
\text { activation } \\
\text { (coherence) } \\
\text { between the verbal- } \\
\text { analytical (T3) and } \\
\text { motor planning (Fz) } \\
\text { regions during a } \\
\text { golf putting task }\end{array}$ \\
\hline $\begin{array}{l}\text { Landers, D. M., } \\
\text { Petruzzello, S. J., } \\
\text { Salazar, W., } \\
\text { Crews, D. J., } \\
\text { Kubitz, K. A., } \\
\text { Gannon, T. L., \& } \\
\text { Han, M., 1991 }\end{array}$ & $\begin{array}{c}\text { The influence of } \\
\text { electrocortical } \\
\text { biofeedback on } \\
\text { performance in pre- } \\
\text { elite archers }\end{array}$ & 24 & $\begin{array}{c}8 \text { women, } \\
16 \text { men }\end{array}$ & $\begin{array}{l}\text { individual: } \\
\text { archery }\end{array}$ & $\begin{array}{l}\text { Arizona State } \\
\text { University, US }\end{array}$ & - & - & & \begin{tabular}{|} 
determine whether \\
EEG biofeedback \\
training could \\
improve archery \\
performance as well \\
as self-reported \\
measures of \\
concentration and \\
self-confidence
\end{tabular} \\
\hline $\begin{array}{c}\text { Landers, D. M., } \\
\text { Han, M., Salazar, } \\
\text { W., Petruzzello, S. } \\
\text { J., } 1994\end{array}$ & $\begin{array}{c}\text { Effects of learning on } \\
\text { electroencephalogra } \\
\text { phic and } \\
\text { electrocardiographic } \\
\text { patterns in novice } \\
\text { archers } \\
\end{array}$ & 11 & $\begin{array}{c}6 \text { women, } \\
5 \text { men }\end{array}$ & $\begin{array}{l}\text { individual: } \\
\text { archery }\end{array}$ & $\begin{array}{l}\text { Arizona State } \\
\text { University, US }\end{array}$ & $\begin{array}{l}19-39 \\
\text { years }\end{array}$ & - & & $\begin{array}{c}\text { Explore whether } \\
\text { hemispheric } \\
\text { asymmetry and } \\
\text { heart rate (HR) } \\
\text { deceleration occur } \\
\text { as a result of } \\
\text { learning } \\
\end{array}$ \\
\hline $\begin{array}{l}\text { Deeny, S. P., } \\
\text { Hillman, C. H., } \\
\text { Janelle, C. M., \& } \\
\text { Hatfield, B. D., } \\
2003\end{array}$ & \begin{tabular}{|c|} 
Cortico-cortical \\
communication and \\
superior \\
performance in \\
skilled marksmen: \\
An EEG coherence \\
analysis \\
\end{tabular} & 19 & men & $\begin{array}{l}\text { individual: } \\
\text { shooting }\end{array}$ & us & \begin{tabular}{|c|} 
experts: \\
$40,8 \pm 15$ \\
years; \\
skilled: \\
$35.6 \pm$ \\
18.8 years \\
\end{tabular} & $\begin{array}{l}\text { international } \\
\text { marksmen }\end{array}$ & & \begin{tabular}{|c|} 
examined whether \\
expert marksmen \\
would exhibit \\
greater \\
autonomy of cortical \\
activation \\
\end{tabular} \\
\hline $\begin{array}{l}\text { Cooke, A., } \\
\text { Kavussanu, M., } \\
\text { Gallicchio, G., } \\
\text { Willoughby, A., } \\
\text { McIntyre, D., \& } \\
\text { Ring, C., 2014 }\end{array}$ & \begin{tabular}{|c|} 
Preparation for \\
action: \\
psychophysiological \\
activity preceding a \\
motor skill as a \\
function of expertise, \\
performance \\
outcome, and \\
psychological \\
pressure
\end{tabular} & 20 & men & $\begin{array}{c}\text { individual: } \\
\text { golf }\end{array}$ & UK & \begin{tabular}{|c|} 
experts: \\
$20,9 \pm$ \\
0,74 \\
years; \\
novices: \\
$19 \pm 0,66$ \\
years
\end{tabular} & - & & $\begin{array}{l}\text { compare cortical, } \\
\text { cardiac, muscular, } \\
\text { and kinematic } \\
\text { activity in } 10 \\
\text { experts and 10 } \\
\text { novices as they } \\
\text { performed golf } \\
\text { putts in low- and } \\
\text { high-pressure } \\
\text { conditions }\end{array}$ \\
\hline $\begin{array}{l}\text { Kleinnijenhuis, } \\
\text { M., Arns, M. W., } \\
\text { \& Rijpma, J., } \\
2008\end{array}$ & \begin{tabular}{|} 
Golf performance \\
enhancement by \\
means of real-life \\
neurrofeedback \\
training based on \\
personalized event- \\
locked EEG profiles
\end{tabular} & 6 & $\mid \begin{array}{c}3 \text { women, } \\
3 \text { men }\end{array}$ & $\begin{array}{c}\text { individual: } \\
\text { golf }\end{array}$ & Vienna, Austria & - & $\begin{array}{l}\text { amateur golf } \\
\text { players }\end{array}$ & & $\begin{array}{c}\text { report on a very } \\
\text { promising, new } \\
\text { method for golf } \\
\text { performance!enhan } \\
\text { cement employing } \\
\text { real-life } \\
\text { neurofeedback } \\
\text { during golf putting. }\end{array}$ \\
\hline $\begin{array}{c}\text { Ming-Yang } \\
\text { Cheng, Kuo-Pin } \\
\text { Wang, Chiao- } \\
\text { Ling Hung, Yu- } \\
\text { Long Tu, Chung- } \\
\text { Ju Huang, Dirk } \\
\text { Koester, Thomas } \\
\text { Schack, Tsung- } \\
\text { Min Hung., 2017 } \\
\end{array}$ & \begin{tabular}{|c|} 
Higher power of \\
sensorimotor \\
rhythm is associated \\
with better \\
performance in \\
skilled air-pistol \\
shooters
\end{tabular} & 24 & $\begin{array}{c}10 \\
\text { women, } \\
14 \text { men }\end{array}$ & $\begin{array}{l}\text { individual: } \\
\text { shooting }\end{array}$ & $\begin{array}{l}\text { National } \\
\text { Taiwan } \\
\text { Sport } \\
\text { University, } \\
\text { Taiwan }\end{array}$ & $\left|\begin{array}{c}18,12 \pm \\
2,39 \text { years }\end{array}\right|$ & \begin{tabular}{|c} 
Level B \\
(According to \\
the \\
International \\
Sports \\
Shooting \\
Federation)
\end{tabular} & & $\begin{array}{c}\text { investigate the } \\
\text { sensorimotor } \\
\text { rhythm power } \\
\text { among intra- } \\
\text { individual trials in } \\
\text { sports, especially for } \\
\text { action execution }\end{array}$ \\
\hline $\begin{array}{l}\text { Sidhu, A., Cooke, } \\
\text { A., } 2021\end{array}$ & $\begin{array}{c}\text { Electroencephalogra } \\
\text { phic neurofeedback } \\
\text { training can } \\
\text { decrease conscious } \\
\text { motor control and } \\
\text { increase single and } \\
\text { dual-task } \\
\text { psychomotor } \\
\text { performance } \\
\end{array}$ & 25 & $\begin{array}{c}16 \\
\text { women, } 9 \\
\text { men }\end{array}$ & - & UK & $\begin{array}{c}\text { females: } \\
23,5 \pm \\
1,37 \\
\text { years, } \\
\text { males: } \\
23,67 \pm 1 \\
\text { years }\end{array}$ & - & & $\begin{array}{c}\text { evaluated whether } \\
\text { electroencephalogra } \\
\text { phic neurofeedback } \\
\text { training can reduce } \\
\text { the adverse effects } \\
\text { of conscious motor } \\
\text { control. }\end{array}$ \\
\hline $\begin{array}{c}\text { Schneider, S., } \\
\text { Askew, C. D., } \\
\text { Diehl, J., Mierau, } \\
\text { A., Kleinert, J., } \\
\text { Abel, T., } \\
\text { Carnahan, H., \& } \\
\text { Strüder, H. K., } \\
\text { 2009 }\end{array}$ & $\begin{array}{l}\text { EEG activity and } \\
\text { mood in health } \\
\text { orientated runners } \\
\text { after different } \\
\text { exercise intensities }\end{array}$ & 24 & $\begin{array}{c}9 \text { women, } \\
15 \text { men }\end{array}$ & $\begin{array}{l}\text { individual: } \\
\text { running }\end{array}$ & $\begin{array}{c}\text { University of } \\
\text { the Sunshine } \\
\text { Coast, Australia }\end{array}$ & \begin{tabular}{|c|} 
females: \\
$32.44 \pm$ \\
9.55 \\
years, \\
males: \\
$28.73 \pm$ \\
6.06 years
\end{tabular} & $\begin{array}{l}\text { amateur } \\
\text { runners }\end{array}$ & & $\begin{array}{c}\text { find changes in EEG } \\
\text { activity and mood } \\
\text { after low, preferred } \\
\text { and high intensity } \\
\text { running }\end{array}$ \\
\hline
\end{tabular}


Vol 4, No 2 (2021): Stadium -Hungarian Journal of Sport Sciences

https://doi.org/10.36439/shjs/2021/2/10557

\begin{tabular}{|c|c|c|c|c|c|c|c|c|c|}
\hline AUTHOR & $\begin{array}{l}\text { TITLE OF THE } \\
\text { STUDY }\end{array}$ & $\begin{array}{l}\text { NUMBER } \\
\text { OF } \\
\text { SUBJECTS }\end{array}$ & GENDER & SPORT & $\begin{array}{c}\text { TERRITORIAL } \\
\text { DEFINITION }\end{array}$ & AGE & CLASS & $\begin{array}{c}\text { METHODOL } \\
\text { OGY }\end{array}$ & AIM OF THE STUDY \\
\hline $\begin{array}{l}\text { Doppelmayr, M.; } \\
\text { Sauseng, P.; } \\
\text { Doppelmayr, H.; } \\
\text { and Mausz, I., } \\
2012\end{array}$ & $\begin{array}{c}\text { Changes in EEG } \\
\text { during Ultralong } \\
\text { Running }\end{array}$ & 1 & men & $\begin{array}{l}\text { individual: } \\
\text { running }\end{array}$ & $\begin{array}{l}\text { Salzburg, } \\
\text { Austria }\end{array}$ & 42 years & - & \multirow{4}{*}{$\begin{array}{c}\text { experimental } \\
\text { procedure }\end{array}$} & $\begin{array}{c}\text { focuse on long- } \\
\text { lasting } \\
\text { exercise during } \\
\text { extended exercise } \\
\text { and under different } \\
\text { thermal } \\
\text { conditions } \\
\end{array}$ \\
\hline $\begin{array}{c}\text { Mechau, D., } \\
\text { Mücke, S., Weiss, } \\
\text { M., \& Liesen, H., } \\
1998\end{array}$ & $\begin{array}{c}\text { Effect of increasing } \\
\text { running velocity on } \\
\text { electroencephalogra } \\
\mathrm{m} \text { in a field test }\end{array}$ & 19 & $\begin{array}{c}2 \text { women, } \\
17 \text { men }\end{array}$ & $\begin{array}{l}\text { individual: } \\
\text { running }\end{array}$ & $\begin{array}{l}\text { University of } \\
\text { Paderborn, } \\
\text { Germany }\end{array}$ & - & $\begin{array}{l}\text { lessure-time } \\
\text { runners }\end{array}$ & & $\begin{array}{c}\text { measure the } \\
\text { electroencephalogra } \\
\text { m (EEG) after } \\
\text { exercise with } \\
\text { increasing intensity }\end{array}$ \\
\hline $\begin{array}{c}\text { Weber, E., \& } \\
\text { Doppelmayr, M., } \\
2016\end{array}$ & $\begin{array}{c}\text { Kinesthetic motor } \\
\text { imagery training } \\
\text { modulates frontal } \\
\text { midline theta during } \\
\text { imagination of a dart } \\
\text { throw }\end{array}$ & 43 & - & $\begin{array}{c}\text { individual: } \\
\text { darts }\end{array}$ & $\begin{array}{l}\text { University of } \\
\text { Salzburg, } \\
\text { Austria }\end{array}$ & \begin{tabular}{|c|} 
KinVis: \\
$24,48 \pm$ \\
4,92 \\
years, \\
Control: \\
$22 \pm 3,43$ \\
years \\
\end{tabular} & - & & $\begin{array}{c}\text { examine motor } \\
\text { imagery dart } \\
\text { training and its } \\
\text { impact on frontal } \\
\text { midline theta activity } \\
\text { (fmt) during MI }\end{array}$ \\
\hline \begin{tabular}{|} 
Faro, H., \\
Machado, D., \\
Bortolotti, H., do \\
Nascimento, P., \\
Moioli, R. C., \\
Elsangedy, H. M., \\
\& Fontes, E. B. , \\
2020
\end{tabular} & $\begin{array}{c}\text { Influence of Judo } \\
\text { Experience on } \\
\text { Neuroelectric } \\
\text { Activity During a } \\
\text { Selective Attention } \\
\text { Task }\end{array}$ & 34 & $\begin{array}{c}5 \text { women, } \\
29 \text { men }\end{array}$ & $\begin{array}{c}\text { individual: } \\
\text { judo }\end{array}$ & Brazil & \begin{tabular}{|} 
black \\
belts: 26.5 \\
\pm 7.9 \\
years, \\
white \\
belts: 25.2 \\
\pm 5.8 \\
years
\end{tabular} & $\begin{array}{l}\text { black belts, } \\
\text { white belts }\end{array}$ & & $\begin{array}{l}\text { compared the } \\
\text { cognitive } \\
\text { performance and } \\
\text { neuroelectric } \\
\text { responses during a } \\
\text { selective attentional } \\
\text { task in judo athletes } \\
\text { with different levels } \\
\text { of expertise. }\end{array}$ \\
\hline $\begin{array}{c}\text { Ludyga, S., } \\
\text { Mücke, M., } \\
\text { Andrä, C., } \\
\text { Gerber, M., \& } \\
\text { Pühse, U., 2021 }\end{array}$ & \begin{tabular}{|} 
Neurophysiological \\
correlates of \\
interference control \\
and response \\
inhibition processes \\
in children and \\
adolescents engaging \\
in open- and closed- \\
skill sports. Journal \\
of sport and health \\
science
\end{tabular} & 184 & - & $\begin{array}{l}\text { open-close } \\
\text { skilled } \\
\text { sports }\end{array}$ & \begin{tabular}{|c|} 
Leipzig, \\
Germany, Basel, \\
Switzerland
\end{tabular} & $9-14$ years & - & $\begin{array}{l}\text { combined } \\
\text { cross- } \\
\text { sectional } \\
\text { data }\end{array}$ & $\begin{array}{c}\text { examine the } \\
\text { association between } \\
\text { regular engagemen } \\
\text { in open- and closed } \\
\text { skill sports and } \\
\text { inhibitory control in } \\
\text { healthy children and } \\
\text { adolescents }\end{array}$ \\
\hline $\begin{array}{l}\text { Villafaina, S., } \\
\text { Castro, M. A., } \\
\text { Pereira, T., } \\
\text { Carvalho Santos, } \\
\text { A., \& Fuentes- } \\
\text { García, J. P., 2021 }\end{array}$ & \begin{tabular}{|c|} 
Neurophysiological \\
and autonomic \\
responses of high \\
and low level chess \\
players during \\
difficult and easy \\
chess endgames - A \\
quantitative EEG and \\
HRV study
\end{tabular} & 28 & - & $\begin{array}{c}\text { individual: } \\
\text { chess }\end{array}$ & Spain & $\begin{array}{c}\text { high: } \\
26,22 \pm \\
12,04 \\
\text { low: } 20,22 \\
\pm 12,25 \\
\text { years }\end{array}$ & high, low level & $\begin{array}{l}\text { cross- } \\
\text { sectional } \\
\text { study }\end{array}$ & $\begin{array}{c}\text { analyze the heart } \\
\text { rate variability } \\
\text { (HRV) and the EEG } \\
\text { power spectrum in } \\
\text { low and high } \\
\text { performance chess } \\
\text { players during easy } \\
\text { and difficult chess } \\
\text { endgames }\end{array}$ \\
\hline $\begin{array}{c}\text { Gosselin, N., } \\
\text { Lassonde, M., } \\
\text { Petit, D., Leclerc, } \\
\text { S., Mongrain, V., } \\
\text { Collie, A., \& } \\
\text { Montplaisir, J., } \\
2009\end{array}$ & $\begin{array}{c}\text { Sleep following sport } \\
\text { related concussion }\end{array}$ & 21 & - & $\begin{array}{l}\text { team: } \\
\text { hockey, } \\
\text { football, } \\
\text { rugby, } \\
\text { soccer, } \\
\text { skating }\end{array}$ & Canada & \begin{tabular}{|c|} 
with \\
concussio \\
n: $24.3 \pm$ \\
6.1 years, \\
control \\
group: \\
$22.6 \pm 2.4$ \\
years \\
\end{tabular} & $\begin{array}{c}\text { professional / } \\
\text { university, } \\
\text { semi- } \\
\text { profesional }\end{array}$ & pilot study & $\begin{array}{c}\text { investigate the } \\
\text { effects of sport- } \\
\text { related concussions } \\
\text { on subjective and } \\
\text { objective sleep } \\
\text { quality }\end{array}$ \\
\hline $\begin{array}{c}\text { Ligeza, T. S., } \\
\text { Nowak, I., } \\
\text { Maciejczyk, M., } \\
\text { Szygula, Z., \& } \\
\text { Wyczesany, M., } \\
2021\end{array}$ & \begin{tabular}{|c|} 
Acute aerobic \\
exercise enhances \\
cortical connectivity \\
between structures \\
involved in shaping \\
mood and improves \\
self-reported mood: \\
An EEG effective- \\
connectivity study in \\
young male adults
\end{tabular} & 20 & men & $\begin{array}{c}\text { individual } \\
\text { and team: } \\
\text { running, } \\
\text { swimming, } \\
\text { weightliftin } \\
\text { g, team } \\
\text { sports, } \\
\text { CrossFit, } \\
\text { cycling, } \\
\text { tennis, } \\
\text { badminton }\end{array}$ & Cracow, Poland & $\begin{array}{c}24.3 \pm 3.4 \\
\text { years }\end{array}$ & $\begin{array}{l}\text { recreational } \\
\text { athletes }\end{array}$ & \multirow[t]{2}{*}{$\begin{array}{c}\text { experimental } \\
\text { procedure }\end{array}$} & $\begin{array}{l}\text { test the } \\
\text { electrophysiological } \\
\text { basis of the } \\
\text { relationship } \\
\text { between exercise } \\
\text { and mood }\end{array}$ \\
\hline $\begin{array}{c}\text { Vogt, T., Kato, K., } \\
\text { Schneider, S., } \\
\text { Türk, S., \& } \\
\text { Kanosue, K., } \\
2017\end{array}$ & \begin{tabular}{|} 
Central neuronal \\
motor behaviour in \\
skilled and less \\
skilled novices - \\
Approaching sports- \\
specific movement \\
techniques
\end{tabular} & 16 & men & $\begin{array}{l}\text { individual: } \\
\text { archery }\end{array}$ & $\begin{array}{c}\text { German Sport } \\
\text { University } \\
\text { Cologne, } \\
\text { Germany }\end{array}$ & $\begin{array}{c}29.69 \pm \\
6.25 \text { years }\end{array}$ & novices & & $\begin{array}{c}\text { examine sports- } \\
\text { specific central } \\
\text { neuronal motor } \\
\text { behaviour during } \\
\text { repetitive voluntary } \\
\text { arrow-releasing } \\
\text { movements in } \\
\text { archery novices }\end{array}$ \\
\hline
\end{tabular}




\section{DISCUSSION}

Electroencephalography (EEG) is a brain imaging technology used since the 1920s. EEG is a relatively inexpensive and easy-to-use tool in the world of research. The primary purpose of this review was to summarize the applications of EEG and its associated neurofeedback in sport. Examine the differences and characteristics of protocols. Assess whether there is this uniform, standardized protocol for each sport and how often it is used among both elite and amateur athletes.

Based on the results of several studies, it can be stated that the decrease in cortical activation is closely correlated with excellent performance and higher levels of intelligence (GRABNER et al., 2006), (NEUBAUER et al., 2004). This phenomenon (neural efficiency) is called the psychomotor efficiency hypothesis (HATFIELD et al., 2020). Experts perform more effectively than novices (BABILONI et al., 2010), (GRABNER et al., 2006).

Task-related performance loss (TRPD) indexes cortical activity (DEL PRECIO et al., 2011). Both low and high TRDP showed lower scores in athletes than in non-athlete control groups. This also confirms the hypothesis of neural efficacy that cortical reactivity decreases when the eyes open at rest (DEL PRECIO et al., 2011).

Due to its physical properties, the use of EEG is limited to sports that require minimal movement and can be easily performed in the laboratory, as a result of which its use in ball games is very limited, despite today's technologies.

Our analysis revealed that in many sports (martial arts, hockey, skating, basketball, snowboarding), sports performance improves as a result of neurofeedback training (MIKICIN et al., 2020). It is important to highlight that despite some research on the topic, the arena of neurotechnology in sports psychology still exists in its rudimentary form and is constrained by a plethora of technological problems (CAMILLE et al., 2020). However, the use of EEG and neurofeedback may be an appropriate tool to develop and study cognitive performance in sport.

The research was supported by: EFOP-3.6.1-16-2016-00022 Debrecen Venture Catapult Programme. 


\section{REFERENCES}

Babiloni, C., Del Percio, C., Iacoboni, M., Infarinato, F., Lizio, R., \& Marzano, N., et al. (2008). Golf putt outcomes are predicted by sensorimotor cerebral EEG rhythms. J. Physiol. 586, 131-139. doi: 10.1113/jphysiol.2007.141630

Babiloni, C., Marzano, N., Infarinato, F., Iacoboni, M., Rizza, G., \& Aschieri, P., et al. (2010). "Neural efficiency" of experts' brain during judgment of actions: a high-resolution EEG study in elite and amateur karate athletes. Behav. Brain Res. 207, 466-475. doi: 10.1016/j.bbr.2009.10.034

Balconi M., Fronda G., \& Vanutelli M. E. (2019a). A gift for gratitude and cooperative behavior: brain and cognitive effects. Soc. Cogn. Affect. Neurosci. 14, 1317-1327. $10.1093 /$ scan/nsaa003

Bazanova, O. M., \& Vernon, D. (2014). Interpreting EEG alpha activity. Neuroscience and biobehavioral reviews, 44, 94-110. https://doi.org/10.1016/i.neubiorev.2013.05.007

Besserve, M., Philippe, M., Florence, G., Laurent, F., Garnero, L., \& Martinerie, J. (2008). Prediction of performance level during a cognitive task from ongoing EEG oscillatory activities. Clinical Neurophysiology,119, 897-908.

Beyer, L., Weiss, T., Hansen, E., Wolf, A., \& Seidel, A. (1990). Dynamics of central nervous activation during motor imagination. International journal of psychophysiology : official journal of the International Organization of Psychophysiology, 9(1), 75-80. https://doi.org/10.1016/0167-8760(90)90008-2

Castermans, T., Duvinage, M., Cheron, G., \& Dutoit, T. (2014). About the cortical origin of the low-delta and high-gamma rhythms observed in EEG signals during treadmill walking. Neuroscience letters, 561, 166-170. https://doi.org/10.1016/j.neulet.2013.12.059

Del Percio, C., Babiloni, C., Marzano, N., Iacoboni, M., Infarinato, F., Vecchio, F., Lizio, R., Aschieri, P., Fiore, A., Toràn, G., Gallamini, M., Baratto, M., \& Eusebi, F. (2009). "Neural efficiency" of athletes' brain for upright standing: a high-resolution EEG study. Brain research bulletin, 79(3-4), 193-200. https://doi.org/10.1016/j.brainresbull.2009.02.001 
Domingos, C., Peralta, M., Prazeres, P., Nan, W., Rosa, A., \& Pereira, J. G. (2021). Session Frequency Matters in Neurofeedback Training of Athletes. Applied psychophysiology and biofeedback, 10.1007/s10484-021-09505-3. Advance online publication. https://doi.org/10.1007/s10484-021-09505-3

Doppelmayr, M., Finkenzeller, T., \& Sauseng, P. (2008). Frontal midline theta in the preshot phase of rifle shooting: differences between experts and novices. Neuropsychologia, 46(5),

1463-1467.

https://doi.org/10.1016/j.neuropsychologia.2007.12.026

Fink, A., Rominger, C., Benedek, M., Perchtold, C. M., Papousek, I., Weiss, E. M., Seidel, A., \& Memmert, D. (2018). EEG alpha activity during imagining creative moves in soccer decision-making situations. Neuropsychologia, 114, 118-124. https://doi.org/10.1016/i.neuropsychologia.2018.04.025

Gao, J., Wang, W., \& Zhang, J. (2016). Explore interregional EEG correlations changed by sport training using feature selection. Computational Intelligence and Neuroscience, 2016, Article 6184823.

García-Monge, A., Rodríguez-Navarro, H., González-Calvo, G., \& Bores-García, D. (2020). Brain Activity during Different Throwing Games: EEG Exploratory Study. International journal of environmental research and public health,17(18), 6796. https://doi.org/10.3390/ijerph17186796

Gardner, F.L., \& Moore, Z.E. (2007). The psychology of enhancing human performance: The Mindfulness-Acceptance-Commitment (MAC) approach. New York: Springe (2) (PDF) Mindfulness and Acceptance Approaches in Sport Performance. Available from:https://www.researchgate.net/publication/266408608 Mindfulness and Accepta nce Approaches in Sport Performance [accessed Apr 29 2021].

Grabner R.H., Neubauer A.C., \& Stern E. Superior performance and neural efficiency: The impact of intelligence and expertise. Brain Res. Bull. 2006;69:422-439. doi: 10.1016/j.brainresbull.2006.02.009.

Hatfield BD, Jaquess KJ, Lo LC, \& Oh H (2020). The cognitive and affective neuroscience of superior athletic performance. In: Tenenbaum G, Eklund RC (eds) Handbook of sport psychology. John Wiley \& Sons, Hoboken, NJ, pp 487-512. 
Janelle C.M., \& Hatfield B.D. (2008). Visual attention and brain processes that underlie expert performance: Implications for sport and military psychology. Military Psychology, 20:39-69. doi: 10.1080/08995600701804798.

Kerick, S. E., Douglass, L. W., \& Hatfield, B. D. (2004). Cerebral cortical adaptations associated with visuomotor practice. Medicine and Science in Sports and Exercise,1, 118129.

Landers, D. M., Han, M., Salazar, W., \& Petruzzello, S. J., et al. (1994). Effects of learning on electroencephalographic and electrocardiographic patterns in novice archers. International Journal of Sport Psychology, 25(3), 313-330.

Lin, C. T., King, J. T., John, A. R., Huang, K. C., Cao, Z., \& Wang, Y. K. (2021). The Impact of Vigorous Cycling Exercise on Visual Attention: A Study With the BR8 Wireless Dry EEG $\begin{array}{llll}\text { System. Frontiers in neuroscience, } & 15,\end{array}$ https://doi.org/10.3389/fnins.2021.621365

Loze, G. M., Collins, D., \& Holmes, P. S. (2001). Pre-shot EEG alpha-power reactivity during expert air-pistol shooting: a comparison of best and worst shots. Journal of sports sciences, 19(9), 727-733. https://doi.org/10.1080/02640410152475856

Mehta, R. K., \& Parasuraman, R. (2013). Neuroergonomics: a review of applications to physical and cognitive work. Front. Hum. Neurosci. 7:889. doi: 10.3389/fnhum.2013.00889

Mikicin, M., Mróz, A., Karczewska-Lindinger, M., Malinowska, K., Mastalerz, A., \& Kowalczyk, M. (2020). Effect of the Neurofeedback-EEG Training During Physical Exercise on the Range of Mental Work Performance and Individual Physiological Parameters in Swimmers. Applied psychophysiology and biofeedback, 45(2), 49-55. https://doi.org/10.1007/s10484-020-09456-1.

Millett, D, Berger, H. (2001). From Psychic Energy to the EEG." Perspectives in Biology and Medicine, vol. 44 no. 4, 522-542. Project MUSE, doi:10.1353/pbm.2001.0070.

Mirifar, A., Beckmann, J., \& Ehrlenspiel, F. (2017). Neurofeedback as supplementary training for optimizing athletes' performance: A systematic review with implications for 
future research. Neuroscience and biobehavioral reviews, 75, 419-432. https://doi.org/10.1016/j.neubiorev.2017.02.005

Muraskin, J., Sherwin, J., \& Sajda, P. (2015). Knowing when not to swing: EEG evidence that enhanced perception-action coupling underlies baseball batter expertise. NeuroImage, 123,1-10. https://doi.org/10.1016/j.neuroimage.2015.08.028

Neubauer A. C., Grabner R.H., Freudenthaler H.H., Beckmann J.F., \& Guthke J. (2004). Intelligence and individual differences in becoming neurally efficient. Acta Psychol., 116:55-74. doi: 10.1016/j.actpsy.2003.11.005.

Németh, K., \& Balogh, L. (2021). THE RELATIONSHIP BETWEEN AROUSAL ZONE, ANXIETY, STRESS AND SPORTS PERFORMANCE. Stadium - Hungarian Journal of Sport Sciences, 3(2), 1-14. https://doi.org/10.36439/SHJS/2020/2/8603

Park, J. L., Fairweather, M. M., \& Donaldson, D. I. (2015). Making the case for mobile cognition: EEG and sports performance. Neuroscience and biobehavioral reviews, 52, 117130. https://doi.org/10.1016/i.neubiorev.2015.02.014

Pereira, M., Argelaguet, F., Millán, J. d. R., \& Lécuyer, A. (2018). Novice shooters with lower pre-shooting alpha power have better performance during competition in a virtual reality scenario. Front. Psychol. 9:527. doi: 10.3389/fpsyg.2018.00527

Perrey, S., \& Besson, P. (2018). Studying brain activity in sports performance: Contributions and issues. Progress in brain research,240, 247-267. https://doi.org/10.1016/bs.pbr.2018.07.004

Pluta, A., Williams, C. C., Binsted, G., Hecker, K. G., \& Krigolson, O. E. (2018). Chasing the zone: Reduced beta power predicts baseball batting performance. Neuroscience letters, 686, 150-154. https://doi.org/10.1016/j.neulet.2018.09.004

Ramsay P. S. (2013). Heinrich Hertz, the father of frequency. The Neurodiagnostic journal, 53(1), 3-26.

Reinecke, K., Cordes, M., Lerch, C., Koutsandréou, F., Schubert, M., Weiss, M., \& Baumeister, J. (2011). From lab to field conditions: a pilot study on EEG methodology in applied sports sciences. Applied psychophysiology and biofeedback, 36(4), 265-271. https://doi.org/10.1007/s10484-011-9166-x 
Salazar, W., Landers, D. M., Petruzzello, S. J., Han, M., Crews, D. J., \& Kubitz, K. A. (1990). Hemispheric asymmetry, cardiac response, and performance in elite archers. Research quarterly for exercise and sport,61(4), 351-359. https://doi.org/10.1080/02701367.1990.10607499

Saleh, G. M., Voyazis, Y., Hance, J., Ratnasothy, J., \& Darzi, A. (2006). Evaluating surgical dexterity during corneal suturing. Archives of Opthalmology, 124, 1263-1266.

Salenius, S., Hari, R. (2003). Synchronous cortical oscillatory activity during motor action. Current Opinion in Neurobiology, vol. 13, no. 6, pp. 678-684, 2003.

Seidel-Marzi, O., \& Ragert, P. (2020). Neurodiagnostics in Sports: Investigating the Athlete's Brain to Augment Performance and Sport-Specific Skills. Frontiers in human neuroscience, 14, 133. https://doi.org/10.3389/fnhum.2020.00133

Shelley-Tremblay, J., Shugrue, J., \& Klein, J. (2006). Changes in eeg laterality index: Effects of social inhibition on putting in novice golfers. Journal of Sport Behaviour, 29, 353-374.

Sidhu, A., \& Cooke, A. (2021). Electroencephalographic neurofeedback training can decrease conscious motor control and increase single and dual-task psychomotor performance. Exp Brain Res 239, 301-313. https://doi.org/10.1007/s00221-020-05935$\underline{3}$.

Symeonidou, E.-R., Nordin, A. D., Hairston, W. D., \& Ferris, D. P. (2018). Effects of cable sway, electrode surface area, and electrode mass on electroencephalography signal quality during motion. Sensors 18:E1073. doi: 10.3390/s18041073 